\title{
LA MARCA PAÍS ESENCIAL COSTA RICA: MÁS ALLÁ DEL BIENESTAR AMBIENTAL
}

\section{THE COUNTRY BRAND ESENCIAL COSTA RICA: BEYOND ENVIRONMENTAL WELFARE}

\author{
Dalia Arce Aguirre* \\ Mónica Ulloa Gómez**
}

Fecha de recepción: $21 / 09 / 2018$

Fecha de aceptación: 23/10/2018

Resumen: El presente trabajo da un panorama sobre lo que es la marca país Esencial Costa Rica. Al ser un sello mundial, se analizan los discursos en torno a su promoción tomando en cuenta que este sello es un atractivo para la apertura económica y para la vinculación con inversores o empresas extranjeras. En el caso costarricense, se puede observar que lo ambiental está acompañado con lo político y económico.

Palabras claves: marca registrada; marca país; economía medioambiental; mercadotecnia; Costa Rica.

Abstract: This paper aims to give an overview of what is the national brand Esencial Costa Rica. Taking into account that it is a global brand, the discourses surrounding its promotion will also be analyzed; taking into consideration that this brand is an attractive way to develop economic opening and to establish links with investors or foreign companies. In that case, it is seen that in the costarrican brand the environmental is link with politics and economics issues.

Keywords: Trade Marks; Country Brand; Environmental Economics; Marketing; Costa Rica.

\section{Introducción}

Desde el año 2011 en Costa Rica, se ha desarrollado un sello país, conocido como Esencial Costa Rica, este ha buscado posicionar a Costa Rica en el nivel internacional como un lugar donde el medio ambiente es un pilar

\footnotetext{
* Costarricense. Bachiller en Historia por la Universidad Nacional, (UNA) Costa Rica. Estudiante de la Maestría en Historia Aplicada, Escuela de Historia, UNA. Correo electrónico: daliaarce65@gmail.com

** Costarricense. Bachiller en Historia por la Universidad Nacional, (UNA) Costa Rica. Estudiante de la Maestría en Historia Aplicada, Escuela de Historia, UNA. Correo electrónico: monicaulloag@gmail.com
} 
fundamental para el desarrollo. La riqueza de su biodiversidad, su cultura y su gente, se vuelven un gran atractivo para el turismo y las empresas transnacionales. A pesar de su creación en el 2011, no fue sino hasta el 2013 cuando la marca se dio a conocer en el nivel internacional, y entró al mercado costarricense en el año 2016. Con solo dos años de ser lanzada al público nacional, la marca ha logrado posicionarse rápidamente, y ha sido adquirida tanto por empresas nacionales como transnacionales. En la actualidad son un total de 259 empresas licenciadas a la marca. Debido al surgimiento de la marca, ha sido de interés analizar el rol ambiental que tienen algunas de esas empresas licenciadas que se respaldan bajo el sello país y si estas en realidad cumplen con parámetros ambientalmente sustentables. A partir de esto, se incursiona en la perspectiva de la ecología política con el fin de comprender las posibles implicaciones medioambientales de esta marca.

\section{Consideraciones teóricas}

El presente trabajo se desarrolló dentro de las bases teóricas de lo que es la ecología política y, por otro lado, la economía ecológica. A partir de la conceptualización de ambas, se planteó la relación con los objetivos que se pretenden abordar en el estudio de la marca país Esencial Costa Rica. En primera instancia es necesario dejar claro cómo fue que la historia ambiental se posicionó dentro de las preocupaciones tanto científicas como sociales. La historia ambiental fue un campo de la historia que se gestó y se comenzó a consolidar desde la década de 1960. De tal modo, según Pasquo, "son abundantes las fuentes que señalan a las primeras manifestaciones de la década de 1960 como las precursoras en anunciar una crisis ambiental. Por aquella década, una variedad de protestas representadas generalmente por jóvenes estaba exigiendo que la naturaleza fuera tratada con respeto (2013) 
Ante un panorama de revoluciones científicas y una explotación desmesurada de los recursos naturales, las sociedades cuestionaron las formas en cómo se obtenían y trataban las materias primas. Fue a partir de dichos cuestionamientos, que las sociedades académicas vieron la necesidad de analizar los fenómenos humanos sobre la utilización de recursos y las consecuencias que estos acarrearon en la naturaleza.

Por otro lado, a partir de las manifestaciones de la década de 1960, cuya preocupación planteó el considerar más objetivamente el ambiente, la crisis ambiental es entendida como el conjunto de:

"Afectaciones en el equilibrio ecológico debido a pérdida de los tres niveles de biodiversidad: genética, de organismos y ecológica porque la actividad extractiva antrópica y la pobre disposición y tratamiento de sus desechos impide la conectividad entre los ecosistemas, especialmente con los modelos contemporáneos de desarrollo" (Jasso, s.f.).

Haciendo referencia nuevamente a la historia ambiental, como una herramienta para intervenir ante los problemas ecológicos mundiales, Suárez postula que:

"Uno de los principales aportes que debiera tener la historia ambiental, es la preocupación por la sustentabilidad o, en las palabras de Guillermo Castro, la historia ambiental debe contribuir a la creación de una geocultura de la sostenibilidad del desarrollo humano, para hacer del Nuevo Mundo de ayer la simiente del mundo nuevo del mañana" (Suárez, 2010).

Desde esos postulados, los fenómenos sociales, económicos y políticos no podían ser analizados con base en el distanciamiento de su entorno natural. De esta manera, "la relación entre naturaleza y sociedad es vista como un proceso 
co-evolutivo donde ambas interaccionan a lo largo del tiempo conformando así un binomio, inseparable, donde la una no se entiende sin la otra" (Suárez, 2010). Por esta razón, entender las problemáticas como una composición entre lo natural y lo social, trajo consigo nuevas formas desde las cuales se proponían soluciones, una de ellas fue la ecología política. Sin embargo, para comprender mejor a qué se refiere ese término, cabe primero explicar qué es la economía ecológica. Sobre esta, la autora Isa Torrealba Suárez menciona que:

"Los pioneros en la economía ecológica son autores del campo de las ciencias sociales y naturales del siglo XIX que se plantearon ciertos aspectos de la relación entre el sistema económico y los flujos de materia y energía, una vez formuladas las leyes biofísicas de la termodinámica, sin embargo, durante la gran parte del siglo XX tal visión se invisibilizó, hasta que logró ser rescatada con impulso -si bien todavía no cuentan con poder- en los 1980 s por lo que denominamos economistas ecológicos modernos quienes sitúan las decisiones en el campo de lo político, lejos de los argumentos teóricos limitados de la economía convencional neoclásica, de la estricta planificación ecológica o, incluso, de la economía ambiental" (Suárez, 2010).

De esa forma, la economía ecológica fue la encargada de dar las primeras luces para que los temas ambientales fueran analizados desde diferentes disciplinas. Para Palacio, "la economía ecológica, se preocupa por cuestionar el olvido sobre la naturaleza en que incurre la economía, que se volvió exclusivamente crematística, es decir de mercado, y que en la economía ambiental es reincorporado como "externalidades" (2006). Este tipo de economía se salió del esquema tradicional e incorporó no solamente las bases económicas, sino que, además, se adaptó a los distintos escenarios donde se llevaba a cabo el desarrollo de las economías, en procura de mantener el bienestar ambiental de estas regiones. 
Como se mencionó anteriormente, este trabajo mantiene como eje central la ecología política. Por las particularidades del sello, la marca país Esencial Costa Rica cumple con las características adecuadas para posicionarse dentro de lo que es la ecología política. Ahora bien, se va a exponer lo que se entiende por ecología política, la cual según Palacio es entendida como:

"El término de ecología política, fue asociado a problemas de acceso y control sobre los recursos y el mal uso de la tierra, y trató de juntar enseñanzas de la ecología con la economía política. Peet y Watts afirman que sus orígenes intelectuales provienen de la fusión entre la cibernética y la teoría de sistemas de Gregory Bateson y Howard Odum; la ecología cultural y antropológica, incluyendo a Julian Steward, Peter Vayda y Roy Rappaport; y los trabajos sobre riesgos y desastres ambientales. Sin embargo, ellos subrayan que las preocupaciones de la economía política cambiaron cualitativamente el asunto, al introducir los temas de pobreza, campesinado, marginalidad y otros conceptos de la economía política crítica. Por regla general, se reconoce a la economía política en las raíces de la ecopol”" (Palacio, 2006).

La ecología política va más allá de los problemas del acceso y el control de los recursos, así como su inadecuado uso. Esta es, además, "una reflexión en torno de las relaciones de poder y saber sobre la naturaleza y su apropiación, transformación, producción y control. Si la ecología a secas pretende ser ciencia, la ecología política es análisis político en torno de la naturaleza" (Palacio, 2006). Si la ecología política analiza la relación entre poder y naturaleza, el proyecto de desarrollo económico de Esencial Costa Rica cumpliría con las pautas necesarias para incluirse dentro de este modelo. Dicho de otro modo, al cumplir con las particularidades de poder que se ejercen sobre

\footnotetext{
${ }^{1}$ Por ECOPOL, entiéndase, ecología política.
} 
la naturaleza, se llega a ver cómo esto "está íntimamente relacionado con procesos sociales y políticos a diversas escalas, y que para entender mejor cualquier problema ambiental es necesario vincular su análisis con las relaciones sociales de producción y la distribución del poder" (CalderónContreras, 2013). Más adelante se explicará esta relación comprendiendo la dinámica de una marca país.

Cabe aclarar que, aunque el estudio de la marca país costarricense no esté fundamentado en los problemas ambientales, sino más bien en la esfera de legitimación de la marca, exponer las distintas aristas que se pueden abordar desde la ecología política, va a permitir tener una visión mucho más amplia de los fenómenos relacionados con el objeto de estudio. Para lo que corresponde estudiar en esta investigación, se tomará el término de la ecología política como una forma de "reflexión política en torno a temas o problemas ambientales" (Palacio, 2006).

Los dos conceptos que se utilizaron para llevar a cabo este análisis se complementan en la medida que:

"La Ecopol reconoce los aportes de la economía política de modo que analiza los procesos de apropiación de la naturaleza, por lo cual revisa su circulación, distribución y consumo. Ello incluye los agentes que participan en ese proceso de elaboración; las formas de su constitución en mercancía desde el punto de vista económico y en "bien" desde el punto de vista jurídico. De ahí se derivan las modalidades y disputas en torno de su apropiación, usufructo y control de la naturaleza. En consecuencia, también analiza las disputas, luchas y negociaciones de esos agentes, lo que deriva en los problemas económicopolíticos de justicia ambiental” (Palacio, 2006).

Gracias a la definición tanto de la economía ecológica como de la ecología política, se pretende dar a conocer los verdaderos intereses de la Marca País de 
Costa Rica. Por esta razón, es necesario visualizarla dentro de estos dos conceptos.

\section{Sobre el método}

\section{Planteamiento de la problemática de investigación}

Esta investigación se planteó a partir de las operaciones llevadas a cabo por la marca país Esencial Costa Rica, en especial cómo ha sido la incorporación de empresas nacionales e internacionales a esta. Para ello, en primera instancia, se han construido los problemas de investigación que guiaron el trabajo, siendo el principal de ellos, ¿de qué manera se ha desarrollado la marca país Esencial Costa Rica dentro del territorio nacional y cuáles han sido sus aportes para el desarrollo económico?

\section{Búsqueda y análisis de fuentes}

Se utilizaron fuentes secundarias como libros y artículos, provenientes de bibliotecas y revistas digitales. La utilización de fuentes primarias como los reglamentos de la marca Esencial Costa Rica fueron fundamentales para comprender la dinámica y las formas de actuar que mantiene la marca. La utilización de fuentes provenientes del Instituto Costarricense de Turismo (ICT) y de otras instituciones gubernamentales ofrecieron material de apoyo para la elaboración del trabajo. Se utilizaron periódicos, cuyo fin era conocer cuál ha sido la difusión de la marca país, dentro de espacios publicitarios y también en los medios de comunicación tanto nacionales como internacionales. El análisis de fuentes centró su atención en conocer cómo la marca ha ido en crecimiento en los últimos dos años. Fue de interés conocer cuál ha sido el impacto ambiental que ha tenido la marca por medio de las empresas que forman parte de ella. Es decir, si realmente el entorno se ha visto afectado por 
el funcionamiento de dichas empresas o si estas han aportado beneficios ambientales o económicos al país.

\section{Presentación y discusión de resultados}

La presentación de los resultados fue dividida en dos partes. La primera albergó los principales resultados y hallazgos de la investigación realizada a partir de la revisión de fuentes. Por otro lado, un segundo segmento, donde se abrió el espacio a la discusión crítica de esos resultados, contemplando las preguntas generadoras de la investigación y los hallazgos en las diferentes fuentes, dando argumentos críticos a esas premisas. Este apartado buscó generar reflexión sobre la verdadera funcionalidad de la marca país, y si en realidad Costa Rica es tan verde como se vende en el extranjero, o su misma estrategia publicitaria ha generado repercusiones dentro de $\mathrm{su}$ medio ambiente.

Para comprender la dinámica de lo que es la marca país costarricense, es necesario dejar claro qué es una marca país, sus principales objetivos y sus proyecciones tanto económicas como ambientales. De esa forma, Granados y Pacheco han conceptualizado la marca país como:

"un factor que puede contribuir a añadir credibilidad a la identidad y a agregar valor a una empresa o marca de producto. Además, puede ser un excelente instrumento para posicionar mundialmente a productos y servicios nacionales, contribuyendo así a la internalización de las pequeñas y medianas empresas que desean competir con el resto de los países en el mercado global. De la misma forma, la Marca País es un instrumento que contribuye al mejoramiento de la economía nacional, y es utilizada como un medio para superar los desafíos del proceso de la globalización y, por lo tanto, facilita una mejor inserción en la economía mundial" (Granados \& Pacheco Revilla, 2007). 
De la misma forma, es considerada como "la herramienta que ayuda a los estados a lograr proyectarse y ser vistos de la manera como ellos deseen, y rescatar las características positivas que tiene el país para hacerlo atractivo y llamativo y, por ende, los productos o servicios originarios de él" (Granados \& Pacheco Revilla, 2007).

Según la reglamentación de Esencial Costa Rica, "la Marca País Costa Rica se constituye en un instrumento estratégico y para la competitividad, mediante la cual se identifica al país, se sella la reputación, se sinergiza la promoción y se genera un posicionamiento diferenciador ante los sectores de exportación, inversiones y turismo" (La Gaceta, 2014). Su principal objetivo es el mejoramiento de la economía nacional en el espacio costarricense e internacional. Esto provoca que la marca país que se fundamenta en una lógica ambiental, ecológica y de conservación, se redireccione a un campo netamente económico. Costa Rica se ha caracterizado y diferenciado gracias a sus espacios políticos y económicos en relación con sus países vecinos, como un espacio idóneo para el desarrollo, es debido a esto que el sello país ha incentivado el discurso político de la Costa Rica verde y desde su utilización ha sido exitoso.

Aunque Esencial Costa Rica tenga sus bases en las características ambientales del país, su objetivo central "es atraer inversión extranjera como fuente alternativa de ingresos al país, adicionales a los obtenidos por turismo" (Aizpurúa, Escudero, Negrete \& Palacios Monti, s.f.). De aquí surge la interrogante, ¿qué tan positiva es la promoción de una Marca País verde, si dentro de sus principales objetivos se apuesta únicamente al factor económico? Ese factor ambiental es el que ha sido aislado y poco visibilizado.

Según los lineamientos de Esencial Costa Rica, dicho sello busca "promocionar de manera integral el turismo, las exportaciones y la inversión extranjera de la mano con la cultura costarricense" (Esencial Costa Rica, s.f.). Por lo tanto, la cuestión ambiental se vuelve un factor secundario dentro de la 
línea discursiva de la empresa. Según Milena Saborío, citada por Villalobos, una marca país "es una estrategia de posicionamiento que busca fortalecer los sectores empresariales, ya sean de turismo, exportaciones e inversión extranjera directa" (2015). Es decir, el interés por la protección ambiental es un "plus" que puede o no ejecutarse mediante la aplicación de este tipo de política económica. A pesar de que este sello vende a Costa Rica como una nación verde, donde su gente y su cultura se vuelven un atractivo para el turismo e inversión, prima el posicionamiento económico que la marca trae consigo para el país.

En el sentido publicitario y de promoción de Esencial Costa Rica, se puede ver mediante distintos medios de comunicación como las instituciones que respaldan este proyecto, principalmente el Instituto Costarricense de Turismo, ha utilizado todos los elementos representativos del país para reflejarlos en el eslogan de la marca, lo cual ha propiciado resultados positivos para la atracción turística, generando mayores ingresos económicos para el país. El reconocimiento del país y su riqueza natural en el ámbito mundial contribuyeron para que la marca país fuera apoyada por distintos sectores, desde las grandes empresas hasta los artesanos costarricenses. De esa forma, el logotipo de una marca país se vuelve trascendental para llamar el interés de posibles inversionistas, así el sello verde de Esencial Costa Rica se encuentra construido bajo la lógica publicitaria, la misma que según Granados y Pacheco describen como:

"Los países deben desarrollar una marca con el propósito de tener un símbolo tangible, elemento distintivo o una representación visual, como por ejemplo un slogan, un símbolo, colores representativos específicos, que ayuden al país a ser promocionados y también que funja como sello diferenciador en el reconocimiento y recordación de los consumidores" (Granados \& Pacheco Revilla, 2007). 
En cuanto a la obtención del sello país, este trae consigo una serie de beneficios para el Gobierno de Costa Rica, el Instituto Costarricense de Turismo y Procomer -sus principales precursores y legitimadores- y, además, para todas aquellas empresas, ya sean nacionales o extranjeras que adquieran la marca. "El licenciamiento de Marca País es una herramienta que busca garantizar la coherencia y coordinación de las actividades e instrumentos de promoción nacional en el exterior, generando paralelamente confianza en los inversionistas y el público extranjero, de manera que se transmita un único mensaje" (NIC Costa Rica, 2018). El discurso de Esencial Costa Rica, no solamente se basa en una "preocupación" por el ambiente, sino que es además un reflejo de la producción y la calidad de la mano de obra nacional. En ese sentido, en la creación de la marca país:

"Lo realmente importante es cómo se crea una identidad de Marca País atractiva, fuerte, coherente y beneficiosa, que permita ser una plataforma para el lanzamiento internacional de la proyección política, económica y cultural de la nación; la internalización de las empresas y marcas propias y un mecanismo promocional importante para la captación de inversiones, atracción de turismo y comercialización de los productos nacionales" (Granados \& Pacheco Revilla, 2007).

Sobre el proceso de licenciamiento, este no demanda una serie de costos económicos que deban adquirir las entidades a quienes se les otorga la marca; "la licencia Esencial COSTA RICA les permite a las empresas utilizar la marca país como sello diferenciador en productos, servicios, materiales corporativos, publicidad, eventos, entre otros. Es importante recordar que PROCOMER no cobra por el uso de la marca país" (Valverde, 2017). Este proceso enmarca 5 grandes puntos, a saber: 


\section{Figura 1 \\ Ejes del proceso de licenciamiento de la marca país Esencial Costa Rica}

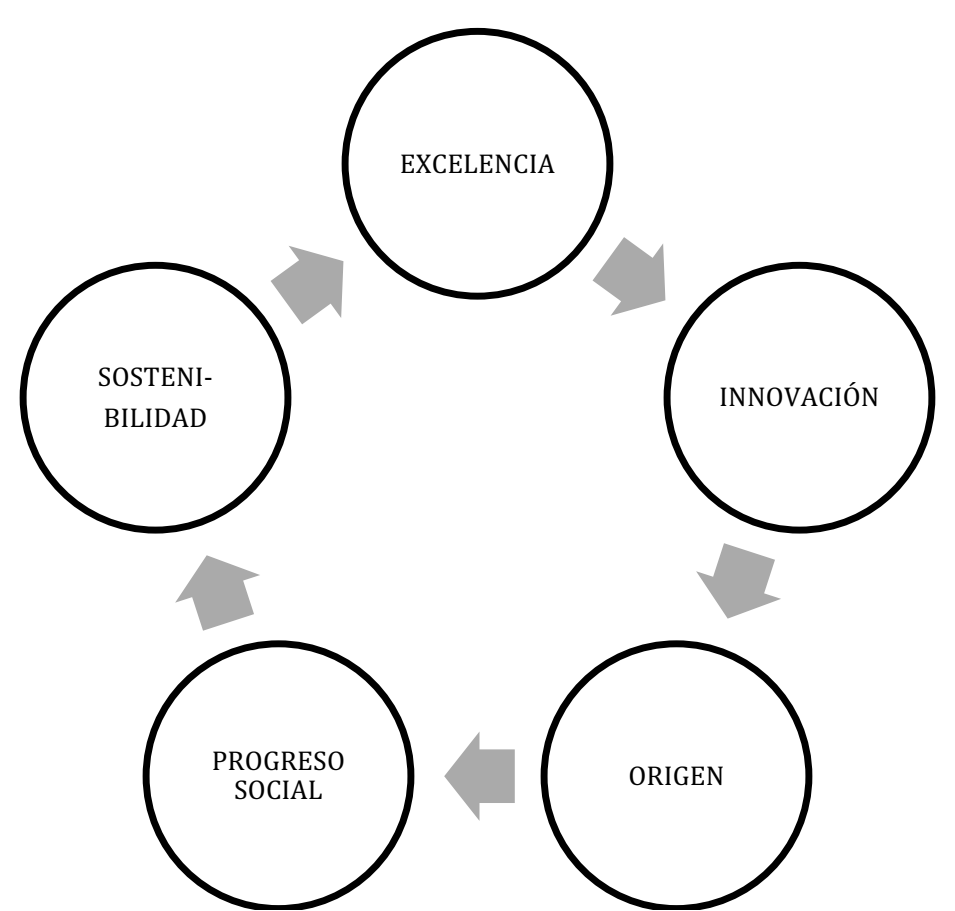

Fuente: Elaboración propia a partir del proceso de licenciamiento de Esencial Costa Rica.

Sobre estos, es importante recalcar el eje de "sostenibilidad", apartado que incluye los impactos ambientales que tienen las empresas licenciadas dentro del país. En este "se miden aspectos como la cultura ambiental, la gestión de la energía, las emisiones, y la gestión del agua” (Pál-Hegedüs, 2017). El protocolo plantea que cada organización o empresa debe comprender que:

"El compromiso ambiental va más allá de la prevención de impactos ambientales adversos por contaminación, también es necesario proteger el entorno natural contra el daño y la degradación producida por las operaciones de la organización. Ahora bien, los objetivos y compromisos que una organización está dispuesta a cumplir deberían ser pertinentes a su contexto e incluir las condiciones ambientales locales o regionales. Estos compromisos 
pueden ser; por ejemplo, la calidad del agua, el reciclaje, la calidad del aire y compromisos relacionados con la mitigación y adaptación al cambio climático, la protección de la biodiversidad y la restauración" (Esencial Costa Rica, s.f.).

Sin embargo, no existe un control adecuado sobre las acciones ambientales llevadas a cabo por las empresas licenciadas con esta marca. Por lo tanto, queda a "libre albedrío" el cumplimiento o no de estas acciones. Esta es una de las mayores preocupaciones sobre el funcionamiento de la marca país, debido a que si dentro de sus pilares se encuentra la sostenibilidad ambiental, sus entes legitimadores deberían mostrarse atentos a que las empresas con el sello Esencial Costa Rica cumplan cada uno de sus lineamientos. Estos compromisos están sujetos a la voluntad de la empresa en tiempo y costos que ellos mismos estimen, de tal forma que no existe una regulación para todas las empresas licenciadas en cuanto a medidas ambientales fomentadas por la marca, factor que en un futuro próximo puede ser perjudicial para el medio ambiente nacional.

A pesar de ello, entre de las instituciones que conforman el sello, destacan algunas que sí han generado beneficios al medio ambiente antes o durante su licenciamiento. Entre estas se encuentra Del Río Exotic Flowers (OBASHE), quien "cuenta con la certificación Rainforest Alliance, que promueve la conservación de la biodiversidad y ecosistemas sostenibles, transformando las prácticas de negocios y uso de tierras" (Esencial Costa Rica, s.f.). Empero, los sellos adicionales que pueden obtener estas empresas, no se ven palpados en resultados concretos dentro de la comunidad o espacio en el que se desarrollen, aportando insumos al mejoramiento de la conciencia ambiental y el medio ambiente como tal.

Por otro lado, es necesario tener en cuenta que Esencial Costa Rica, no responde únicamente a cuestiones de promoción o preservación ambiental. Por 
su parte, también en el desarrollo y respaldo de la marca se puede ver que el talento humano y la producción de bienes, a raíz de este, son los resultados que se propuso la marca para aumentar la atracción extranjera y las inversiones en el país. Así es como se puede ver la confrontación entre las preocupaciones que genera el impacto ambiental y, por otro lado, las preocupaciones económicas.

\section{Discusiones relevantes}

La marca país, es una estrategia económica que ha utilizado Costa Rica para proyectarse en el extranjero. De esa forma uno de sus principales objetivos es "expresar una idea central, articulada y consistente del país, diferenciándolo de sus competidores. Esto es clave para potenciar la gestión de atracción de inversión extranjera directa, la promoción de las exportaciones y el turismo" (Embajada de Costa Rica, 2013). Con el afán de venderse como un país que reúne las condiciones necesarias ha echado mano de factores nacionales particulares, tales como la cultura, su gente y especialmente su ambiente, tres pilares fundamentales en toda la creación y desarrollo de la marca.

El factor ambiente ha sido tomado para vender la imagen costarricense ante el mundo, como un país verde y ecológico. Empero, a pesar de que sus lineamientos indican el compromiso ambiental que tienen tanto la marca como las empresas licenciadas en el tema ambiental, se ven reflejadas carencias en el control de cumplimiento de estos estándares que se observan desde la redacción de los protocolos.

Gonzalo Vilar, Director de Estrategia de Bloom Consulting, indicó que "de acuerdo con la data analizada en las dimensiones de turismo, inversión y comercio, la marca país de Costa Rica está bien establecida, goza de una excelente reputación a nivel mundial, una mejor percepción que los competidores analizados en la zona y un sentimiento de positivismo hacia ella” 
(Mora, 2017). Sin embargo, no se está analizando la magnitud del impacto ambiental o los recursos utilizados por las empresas en cuanto a la elaboración de sus productos, lo primordial es que estos cuenten con un alto estándar de calidad y posicionamiento económico.

El tema ambiental si bien es cierto ha sido muy señalado en la teoría por parte de la marca, en la práctica existen inconsistencias entre lo que se debería aplicar y lo que en realidad sucede. Esto último en especial por las molestias de algunos sectores sociales en torno a los problemas ambientales que las marcas registradas ocasionan en el medio ambiente. Aunque las empresas están obligadas a contar con sellos de calidad para poder ser licenciadas, no indica que cumplan con los requerimientos sobre impacto ambiental.

En Costa Rica todavía falta que se realicen estudios en torno al impacto ambiental que genera la fomentación de una marca país. En la actualidad solo se conoce la realización de un estudio de percepción sobre el impacto internacional de la marca, según el periódico La Nación. Es necesario que se tome en consideración a las comunidades donde están instaladas dichas organizaciones para conocer el impacto inmediato que conllevan las operaciones de estas instituciones. El país debe ser crítico de los pros y contras que la marca país está representando en el medio ambiente nacional, partiendo de la premisa que la implementación de esta responde a criterios económicos propiamente dichos.

Por otro lado, el discurso y la promoción de la marca país es espectacular en cuanto a producción y alcance. Solo para hacerse una idea, en el año 2016, "la marca país ha tenido un gran acercamiento con el público nacional bajo el lema "La esencia sos vos", lo que ha dado como resultado que cada día hay más empresas de distintos sectores y tamaños interesadas en licenciarse y ser parte de este proyecto país que se construye entre todos" (Arias, 2016). Esto quiere decir que dentro de la promoción del sello país, se muestran los mejores 
paisajes y la cantidad de recursos que tiene Costa Rica para ser explotados o mejorados. La marca Esencial Costa Rica es el resultado "del trabajo entre el sector público y el privado; y con la que busca ser reconocido como un destino que ofrece belleza natural y una industria de tecnología e innovación y, además, con capacidad de exportar productos de calidad" (PROESA, 2014). Con esto, se ha logrado que:

"essential Costa Rica: My Choice, Naturally sea una campaña sensorial, inspiradora y aspiracional, que muestra a Costa Rica como un destino generador de experiencias auténticas de viaje, que crean en el visitante un sentimiento de bienestar, porque se trata de un mensaje directo, personal y motivador" (Imagazinetur, s.f.).

Aún queda mucho por hacer cumplir los estatutos del reglamento de Esencial Costa Rica. Es una tarea que no corresponde únicamente a las instituciones que promueven la adquisición de la marca, sino que también se debe concientizar a las empresas que llevan su sello y respaldo para que el crecimiento económico vaya de la mano con el buen manejo ambiental. La forma en cómo se desarrollan las marcas país es con la finalidad de proporcionar tanto a los sectores ecológicos como económicos un mejoramiento. Sin embargo, en muchas ocasiones se dejan de lado los lineamientos que respaldan la marca, y esto trae con ellos una serie de problemas, los cuales radican principalmente en la negligencia a la hora de medir los impactos causados sobre la naturaleza por y para la producción de bienes.

Con estas referencias sobre el sello país, debería analizarse con mayor cuidado y detenimiento el verdadero valor que tiene una de sus bases: la sostenibilidad. Tal cual se propone que "la sostenibilidad para la Marca País es una meta viva, un camino permanente, hacia el futuro y la unión entre la 
gestión ambiental, social y financiera" (La Gaceta, 2014). Para que Esencial Costa Rica tenga un éxito real, no se debe encerrar solamente en lo económico, sino poner los ojos en lo ambiental, que finalmente contribuye como parte de un todo al desarrollo de un país.

\section{Conclusiones}

La marca país Esencial Costa Rica nació bajo la premisa de proyectar a Costa Rica en el extranjero como una república con un alto porcentaje de posibilidades de inversión económica, utilizando factores como su cultura, su gente y su naturaleza. A pesar de que el factor ambiente, se encuentra presente en sus diferentes discursos y promociones, ha sido en realidad un efecto "secundario" de su accionar. Aunque se cuenta con los protocolos donde se establece la importancia del cuido del medio ambiente, el grupo Esencial Costa Rica no realiza un control eficaz del cumplimiento por parte de las empresas.

El turismo actualmente es uno de los ingresos económicos más significativos del país. Por este motivo, es que se ha apoyado sobre medida a este sector; con ello, el desarrollo y éxito de la marca país Esencial Costa Rica se ha podido fortalecer desde su creación en el 2013. Gracias a las herramientas publicitarias, y a la marca, muchos espacios de potencial turístico e incluso productos nacionales se han respaldado bajo Esencial Costa Rica, lo cual los ha beneficiado para obtener un cierto reconocimiento, y así potenciar sus empresas o productos.

Cabe resaltar que también "a través de la estrategia de Imagen de Marca País, las PYMES se ven comprometidas a transformarse y mejorar, de manera continua, sus servicios y su producción para utilizar el logo. Es decir, la Marca País garantiza estándares de calidad a los consumidores con los cuales las empresas se deben comprometer para no fallarle ni al país, ni a la marca, ni 
a su compañía” (Granados \& Pacheco Revilla, 2007). Se podría considerar que la marca no únicamente se direcciona a la inversión extranjera, sino que también trata de potenciar la economía nacional desde sus propios productores.

Por su parte, grupos de movimientos ambientales han expresado la molestia por utilizar esta herramienta de mercadeo, en la cual se muestra solamente lo verde del país. Se ha apelado en estos últimos años, que la marca no se preocupa por el bienestar ambiental del país, pues muchas de las empresas licenciadas a ella son instituciones que han generado impacto negativo en el medio ambiente. El discurso ambientalista que se utiliza ha sido una estrategia de publicidad para atraer la inversión extranjera de grandes transnacionales. Además, la idea de que Esencial Costa Rica pertenece únicamente al país es un discurso falso, ya que dentro del sello país se incluyen empresas transnacionales, las cuales tienen el beneficio de obtener la marca por medio del cumplimiento de los requisitos.

Por lo tanto, debe ser necesario darles seguimiento a las acciones llevadas a cabo por parte de la marca país, esto con el fin de medir las acciones de seguimiento a las empresas licenciadas en procura de la ejecución de los requerimientos solicitados. Debe primar el interés por la protección del medio ambiente, de forma que la economía, la política y el ambiente puedan interactuar homólogamente en un espacio de cooperación como el que se desea producir por medio de la marca país Esencial Costa Rica. 


\section{Referencias}

"El uso de la marca país 'esencial Costa Rica' exige a las empresas una nota alta en excelencia”. (2014, 8 de enero). La Nación, URL: https://www.nacion.com/economia/negocios/el-uso-de-la-marca-paisesencial-costa-rica-exige-a-las-empresas-una-nota-alta-enexcelencia/PYTLCGI2TJHBNMWDTX6VMBLBYE/story/

"País evaluará el impacto de la marca 'Esencial Costa Rica". (2017, 20 de marzo). La Nación, URL: https://www.nacion.com/economia/negocios/pais-evaluara-el-impacto-dela-marca-esencial-costarica/MY6WMY5KCVFNJHFYAGJYFF42GE/story/

Aizpurúa, S., Escudero, E., Negrete, D., \& Palacios Monti, C. (s.f.). Imagen Empresaria. Argentina: Universidad de Palermo.

Arias, J. P. (2016, 21 de julio). "189 empresas ya tienen marca Esencial Costa Rica". CRHoy.com, URL: http://www.crhoy.com/archivo/189-empresasya-tienen-marca-esencial-costa-rica/economia/

Calderón-Contreras, R. (mayo-agosto, 2013). "Ecología política: Hacia un mejor entendimiento de los problemas socioterritoriales". Economía, Sociedad y Territorio, 13(42), 561-569, disponible en URL: http://www.redalyc.org/articulo.oa?id=11126608009

Chandeck, E. (2016, 8 de abril). "Marca País 'esencial COSTA RICA' una herramienta de promoción". Baker Tilly Costa Rica, URL: http://www.bakertillycostarica.com/marca-pais-esencial-costa-rica-unaherramienta-de-promocion/

Díaz, R. (2017, 29 de agosto). "Esencial Costa Rica se posiciona en el mundo". La República, URL: https://www.larepublica.net/noticia/esencial-costarica-se-posiciona-en-el-mundo

Embajada de Costa Rica. (2013). embajadadecostarica.org, URL: https://www.embajadadecostarica.org/index.php?option=com content\&vi $\underline{\text { ew }=\text { article\&id }=105 \& \text { Itemid }=553}$

Esencial Costa Rica. (s.f.). esencial COSTA RICA. URL: https://www.esencialcostarica.com/ 
Forbes, R. (2015). "Progreso social en la Marca País Esencial Costa Rica". CEGESTI: Éxito Empresarial.

Granados, M. C., \& Pacheco Revilla, M. (2007). La Marca Paiís como un instrumento para contrubuir al proceso de internalización de las PYMES. San José: UNED.

Imagazinetur. (s.f.). "ICT lanzó al mundo essential Costa Rica. My choice, Naturally".

http://www.imagazinetur.com/modules/news/article.php?storyid=3868

Jasso, G. M. (s.f.). "Historia Ambiental. Una disciplina necesaria para la educación ambiental y las ciencias ambientales". UASLP.

Mora, A. (2017, 7 de julio). "Marca País Esencial COSTA RICA es la que más ha crecido en el continente". elpais.cr., URL: https://www.elpais.cr/2017/07/07/marca-pais-esencial-costa-rica-es-laque-mas-ha-crecido-en-el-continente/

Mora, E. F. (2017, 6 de julio). "Estudio Marca País Esencial Costa Rica es la de mayor crecimiento en América". El Financiero, URL: https://www.elfinancierocr.com/negocios/estudio-marca-pais-esencialcosta-rica-es-la-de-mayor-crecimiento-enamerica/OTDB3UV62FHRBGKBRC2SMRB4OU/story/

NIC Costa Rica. (2018, 9 de abril). “.cr es orgullosamente Esencial Costa Rica" URL: https://www.nic.cr/ver-noticia/114/cr-es-orgullosamente-esencialcosta-rical

La Gaceta. (2014, 25 de marzo).

Pál-Hegedüs, D. (2017, 17 de noviembre). "Marca País 'Esencial Costa Rica' se posiciona". $E l \quad$ Financiero, https://www.elfinancierocr.com/opinion/marca-pais-esencial-costa-rica-seposiciona/35KVAFT7SFB2LB4VO6PSGRGVKA/story/

Palacio, G. A. (2006). "Breve guía de introducción a la ecología política (Ecopol): origenes, inspiradores, aportes y temas de actualidad". Gestión y Ambiente, 9(3), 7-20, URL: https://revistas.unal.edu.co/index.php/gestion/article/view/49672 
Pasquo, F. D. (2013). "Una historia de la problemática ambiental y de sus efectos sobre la ecología disciplinar". Scientle Studia, 11(3), 557-581, disponible en URL: http://www.scielo.br/pdf/ss/v11n3/06.pdf

PROESA (Organismo Promotor de Exportaciones e Inversiones de El Salvador). (2014, 7 de noviembre). "La marca país impacta en el desarrollo socioeconómico de una nación (IV): esencial COSTA RICA". URL: http://www.proesa.gob.sv/novedades/noticias/item/706-marca-paisesencial-costa-rica

Rojas, M. (2016, 14 de septiembre). "Costa Rica, una marca país de qué". Seminario Universidad, URL: https://semanariouniversidad.com/opinion/costa-rica-una-marca-pais/

Saborío Villalobos, M. (2015). "Marca país esencial Costa Rica: herramienta para la competitividad". CEGESTI: Éxito Empresarial, (284), disponible en http://www.cegesti.org/exitoempresarial/publicaciones/publicacion_284_2 $\underline{00415 \text { es.pdf }}$

Suárez, I. T. (2010). Sustentabilidad, Historia ambiental y trandisciplinariedad. Liberia, Guanacaste, Costa Rica: CIAHC.

Valverde, D. (2017, 4 de agosto) "Con Esencial Costa Rica hacemos realidad nuestra promesa al mundo". America-economia-ca.com, URL: http://americaeconomia-ca.com/2017/08/04/con-esencial-costa-ricahacemos-realidad-nuestra-promesa-al-mundo/ 